\title{
IMPLEMENTATION ASPECTS AND OFFLINE DIGITAL SIGNAL PROCESSING OF A SMART PEBBLE FOR RIVER BED SEDIMENT TRANSPORT MONITORING
}

\author{
Nihal Kularatna, Senior Member \\ Dept of Engineering, School of Science and Engineering \\ The University of Waikato \\ Private Bag 3105, Hamilton 3240, New Zealand \\ Email:n.kularatna@auckland.ac.nz
}

\author{
Bruce Melville ${ }^{1}$, Ehad Akeila ${ }^{2}$, Dulsha Kularatna ${ }^{3}$ \\ ${ }^{1}$ Dept of Civil and Environmental Engineering ${ }^{2,3}$ Dept of \\ Electrical and Computer Engineering \\ University of Auckland, New Zealand \\ ${ }^{1}$ Email:b.melville@auckland.ac.nz
}

Abstract - Conceptualization aspects of a smart sediment particle (smart pebble) for monitoring of sediment transport in riverbeds have been documented previously[1]. However, this mixed signal approach was done only at a conceptual level and lacks complete implementation aspects such as limited PCB real estate, a miniaturized power source and adequately addressing the offset errors. A fully digital version with multiple strap-down MEMs, signal conditioning blocks, an 8-bit processor and a memory subsystem was designed and assembled within a less than $4 \mathrm{~cm}$ diameter sphere to allow data capture for up to $\mathbf{1 5}$ minutes. This compact subsystem allows exporting of output data, stored within the memory from nine sampled MEM sensors, into an offline-processing environment for further processing to generate essential motion information. Complex mathematical algorithms for axis conversion, etc, are housed within the offline-processing environment reducing the burden on the smart pebble. The total electronic subsystem embedded within the pebble together with the external processing algorithms tackle cumulative errors, gravitational compensation requirements and offset errors, while being powered by a specially designed power stage, based on a single alkaline cell.

\section{INTRODUCTION}

Low cost MEMS for consumer and automotive applications have been used in advanced engineering applications. [2] and [3] indicate that low cost MEMS are usable in a strap-down inertial platform, if the cumulative errors are managed with adequate accuracy. The success of a proof of concept project [1], based on a mixed signal approach, proved recently that a "smart" sediment particle could provide significant insight into the process of sediment entrainment, which would impact upon diverse fields including river flooding.
The granular material (sands and gravels) comprising the beds of rivers is typically in motion during periods of high river flow and stationary at other times. One of the most intriguing problems of this sediment transport process is the cause of the initial motion, i.e. the "entrainment" or pickup, of individual sediment grains. The smart sediment particle will be capable of recording its own motion in response to a range of aspects of its immediate surroundings, specifically the local water flows and the influence of the neighboring sediment particles. It will then be especially useful for elucidation of the entrainment process. Simultaneous measurement of the very complicated turbulent water flow will also be made.

Figure 1 depicts the test arrangement for use of the smart sediment particle in experiments designed to elucidate the sediment entrainment process.

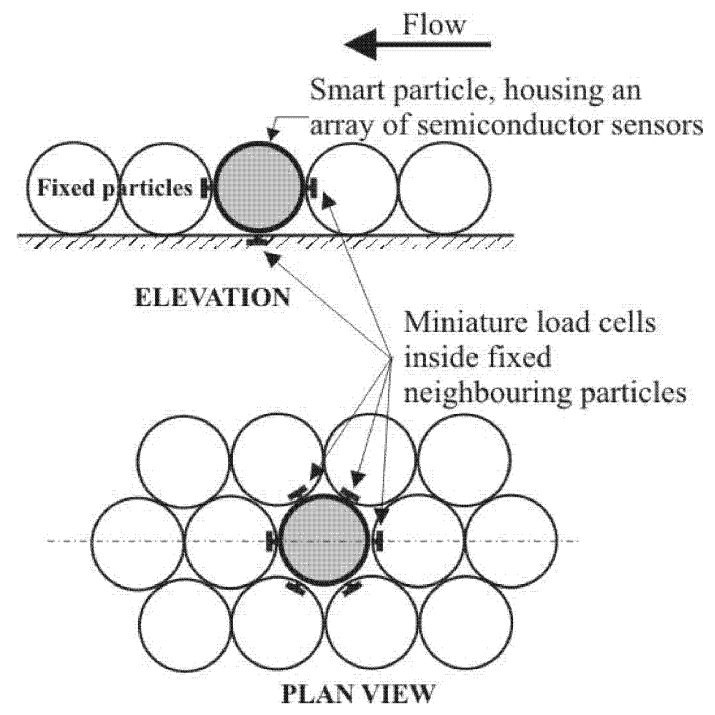

Figure 1: Test arrangement using the smart sediment particle 
As shown, the smart particle will be positioned amongst a bed of similar (fixed) particles. Miniature load cells will be used to measure the inter-particle forces between the smart particle and its neighboring fixed particles. The measurements of the motion of the smart particle, the interparticle forces, and the flow turbulence will be used to develop a conceptual model of the entrainment process. The conceptual model will represent a most significant advance in understanding of the process of sediment transport.

In developing a smart pebble for this special application, the following key features were essential in addition to the basic motion-related parameter monitoring capability: (i) the physical dimensions of the pebble should be only 3 to $4 \mathrm{~cm}$ in diameter; (ii) it should operate underwater, with its specific gravity close to natural pebbles; and (iii) it should be self contained with an energy source, memory and the ability to determine its location.

Therefore, key design considerations are packaging, powering, relative physical placement of the MEMS, signal conditioning and providing a bare minimum digital subsystem and a compact and simple data communication interface. The design then must allow housing of a multiple number of multiplexed data converter ICs with a fair amount of signal conditioning within a $3 \mathrm{~cm}$ diameter pebble. Given the application requirement, where only low frequency components are required (motion parameters $<20 \mathrm{~Hz}$ ), with an accuracy in the range of $2-7 \%$, a key objective is to minimize volume and energy consumption. This suggests the use of a lithium or alkaline cell together with charge pump circuits etc, together with a miniature digital processing subsystem based on commercial off-the-shelf (COTS) components, while moving almost all complex digital signal processing algorithms to an offline platform. Using video recording and image analysis and sensor calibration techniques, semi-processed motion information can be analyzed with adequate accuracy for the riverbed motion monitoring.

\section{MATHEMTICAL CONCEPTS}

Inertial navigation system (INS) concepts [6] are used as the basis of this work. An INS is a system, which can determine its exact location in three-dimensional space, relative to a known starting location. The location is resolved by integrating the accelerations and rotations about the three axes. Three accelerometers and three gyroscopes measure the accelerations and angular rotations about the three axes, giving the particle six degrees of freedom. The reference frame consisting of three axes of the body of the pebble is known as the Body Frame. The set of sensor modules, organized in orthogonal form, measure the accelerations and gyrations with reference to the body frame. As the forces acting on the pebble need to be measured relative to a stationary frame of reference a conversion is necessary. There are several methods of performing a coordinate transformation [6]. The Euler Angle method [7] was chosen as the most appropriate method, given the processing and time constraints in this project.
The Euler Angle method performs the coordinate transformation as three rotations about the three axes. To transform the body frame related motion parameters to the reference frame, three angular rotations, $\psi, \theta$ and $\varphi$ are performed around the $\mathrm{z}, \mathrm{y}$ and $\mathrm{x}$ axes, respectively. When the three rotations are performed as per Figure 2, we get three matrices $\mathrm{C}_{1}, \mathrm{C}_{2}, \mathrm{C}_{3}$, representing the rotations as below:

Rotation $\psi$ about the reference $\mathrm{Z}$ axis,

$C_{1}=\left[\begin{array}{ccc}\operatorname{Cos}(\psi) & \operatorname{Sin}(\psi) & 0 \\ -\operatorname{Sin}(\psi) & \operatorname{Cos}(\psi) & 0 \\ 0 & 0 & 1\end{array}\right]$

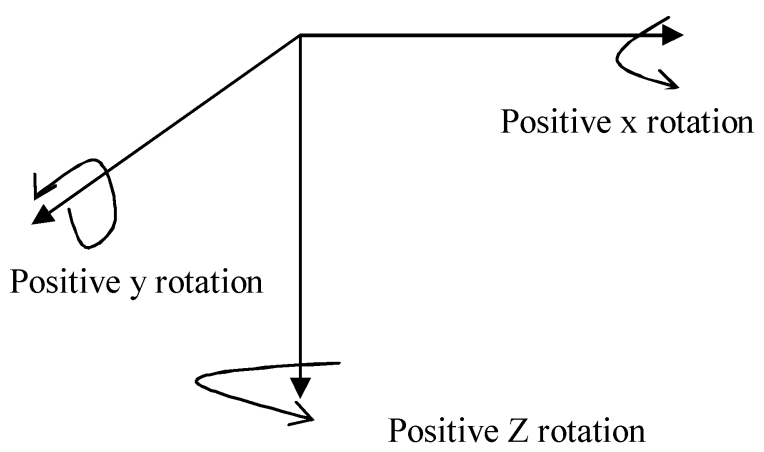

Figure 2: Axis conversion from body frame to reference frame

Rotation $\theta$ about the new $y$-axis,

$$
C_{2}=\left[\begin{array}{ccc}
\operatorname{Cos}(\theta) & 0 & -\operatorname{Sin}(\theta) \\
0 & 1 & 0 \\
\operatorname{Sin}(\theta) & 0 & \operatorname{Cos}(\theta)
\end{array}\right]
$$

Rotation $\varphi$ about the new x-axis,

$$
C_{3}=\left|\begin{array}{ccc}
1 & 0 & 0 \\
0 & \operatorname{Cos}(\varphi) & \operatorname{Sin}(\varphi) \\
0 & -\operatorname{Sin}(\varphi) & \operatorname{Cos}(\varphi)
\end{array}\right|
$$

The three individual matrices can be combined into one complete matrix as follows:

$$
C_{n}^{b}=C_{3} \bullet C_{2} \bullet C_{1}
$$

The complete matrix $C_{n}^{b}$ is known as the rotation matrix [6]. 
The conversion of any vector from the body frame to the reference frame is achieved by the multiplication of the body frame vector by the transpose of the rotation matrix. This is indicated in the following:

$$
r^{n}=C_{b}^{n} \bullet r^{b}
$$

Where,

$r^{n}$ is the vector in reference frame,

$C_{b}^{n}$ is the transpose of the rotation matrix $C_{n}^{b}$, and

$r^{b}$ is the vector in the body frame.

For the order of rotation in Equation (5) the transpose of the rotation matrix $C_{n}^{b}$ is as follows [6].

$C_{b}^{n}=\left[\begin{array}{ccc}\operatorname{Co}(\theta) \operatorname{Co}(\psi) & -\operatorname{Cos}(\varphi) \operatorname{Sin}(\psi)+\operatorname{Sin}(\varphi) \operatorname{Sin}(\theta) \operatorname{Co}(\psi) & \operatorname{Sin}(\varphi) \operatorname{Sin}(\psi)+\operatorname{Cos}(\varphi) \operatorname{Sin}(\theta) \operatorname{Cos}(\psi) \\ \operatorname{Co}(\theta) \operatorname{Sin}(\psi) & \operatorname{Co}(\varphi) \operatorname{Cos}(\psi)+\operatorname{Sin}(\varphi) \operatorname{Sin}(\theta) \operatorname{Sin}(\psi) & -\operatorname{Sin}(\varphi) \operatorname{Co}(\psi)+\operatorname{Co}(\varphi) \operatorname{Sin}(\theta) \operatorname{Sin}(\psi) \\ -\operatorname{Sin}(\theta) & \operatorname{Sin}(\varphi) \operatorname{Co}(\theta) & \operatorname{Co}(\varphi) \operatorname{Cos}(\theta)\end{array}\right]$

The above rotation matrix is quite complex and a direct implementation of the same in a DSP subsystem with suitable angular inputs on fast sampling basis is a tedious task and the development time could be excessive. Also with the proof of concept project[1] where a simplified mixed signal approach was used it was ascertained that reasonably acceptable accuracies could be achieved based on the following special attributes of the civil engineering problem.

I. The need for short duration of data acquisition, usually less than 30 minutes.

II. The pebble is expected to monitor motion parameters with frequency components within about $20 \mathrm{~Hz}$.

III. Autonomous underwater operation would be required.

IV. The pebble was expected to have the correct specific gravity (about 2.65).

V. Digitally acquired and stored raw data could be easily processed by a program like Matlab.

In developing a simplified prototype, one important approximation used for simplifying the mathematics involved in the coordinate transformation was the case of $[\operatorname{Sin} \mathrm{x} \approx \mathrm{x}]$ and similar trigonometric relationships. In such a situation, if commercial off the shelf (COTS) accelerometers and gyroscope ICs are used to monitor instantaneous motion parameters, $r^{b}$ vector can be indicated as,

$$
r^{b}=\left[\begin{array}{c}
A_{x-b} \\
A_{y-b} \\
A_{z-b}
\end{array}\right]
$$

where $A_{x-b}, A_{y-b}$ and $A_{z-b}$ are the body frame accelerations in the corresponding axes. With relatively fast sampling and the angular rotations during the sampling periods being small, $\psi, \theta$ and $\varphi$ will be small enough to use suitable trigonometric approximations. In such a case,

$$
C_{b}^{n}=\left[\begin{array}{ccc}
1 & -\psi & \theta \\
\psi & 1 & \phi \\
-\theta & \phi & 1
\end{array}\right]
$$

This gives the simplified relationship for the $r^{n}$ vector in the reference frame as,

$$
r^{n}=\left[\begin{array}{ccc}
1 & -\psi & \theta \\
\psi & 1 & \phi \\
-\theta & \phi & 1
\end{array}\right]\left[\begin{array}{c}
A_{x-b} \\
A_{y-b} \\
A_{z-b}
\end{array}\right]
$$

For the angular rotation parameters, the following relationships were used.

$$
\psi=\int \omega_{z} d t, \theta=\int \omega_{y} d t, \phi=\int \omega_{x} d t
$$

where $\omega_{x}, \omega_{y}$ and $\omega_{z}$ are the gyrations in respective axes.

Based on these mathematical relationships, it was decided to have a simple and energy efficient basic motion sensor system. Limited digital blocks were to be used to store A to D converted data, which were to be exported to a suitably developed Matlab program initially. At the second stage of the project, a low power DSP subsystem is to be used to have fully axis converted information within the pebble itself.

\section{IMPLEMENTAION}

In order to monitor the accelerations, COTS type accelerometers and gyroscope MEMS from Analog Devices were considered based on their sensitivity, accuracy, noise behavior and the offset as well as the total cost of the system. ADXL202 dual axis accelerometers and ADXRS150 yaw rate gyroscopes were found suitable. These devices were placed in three orthogonal sensor modules with suitable signal conditioning circuitry. The total system including off line processing is shown in Figure 3. Figure 4 depicts the block diagram of the system. 


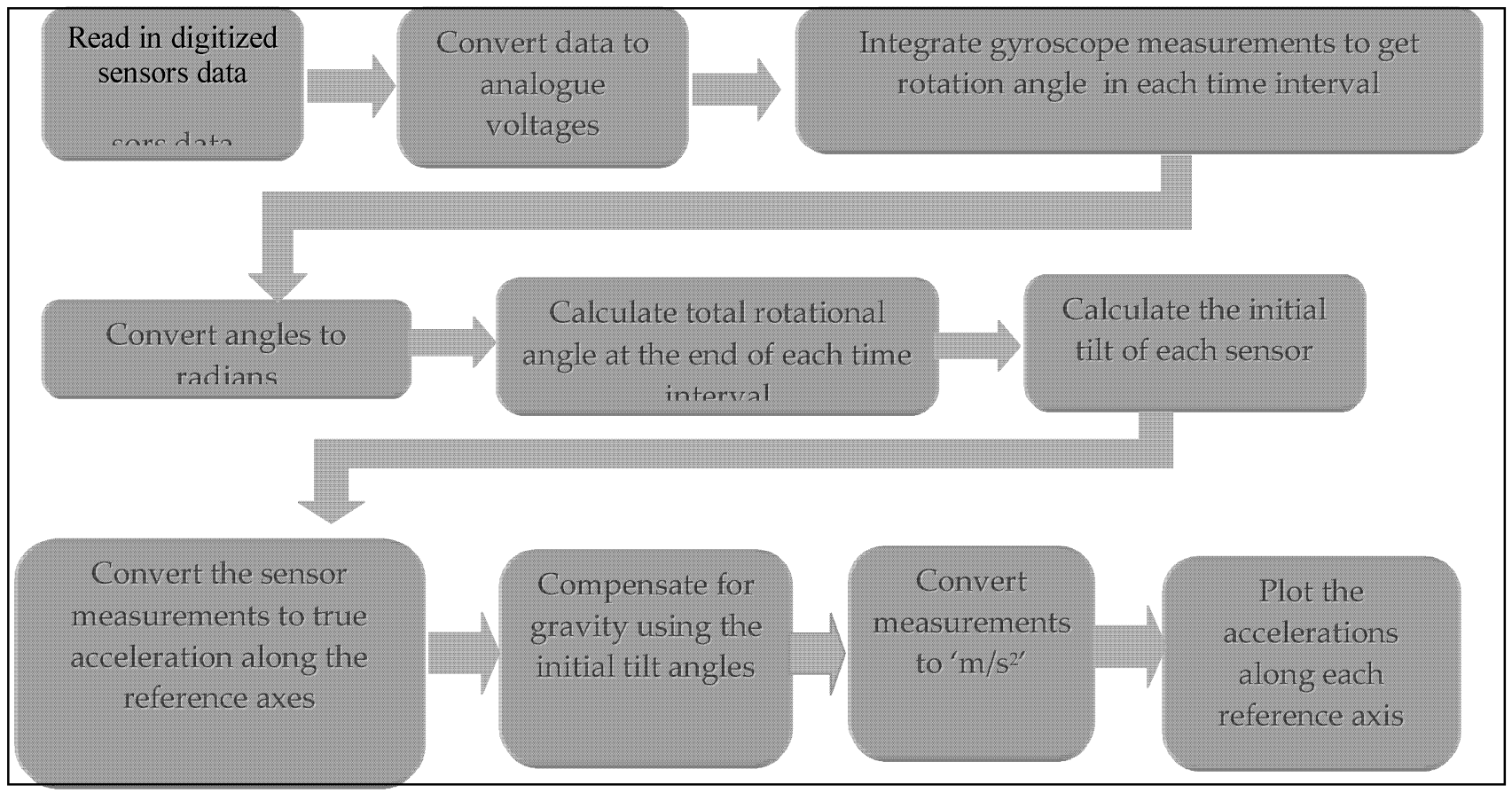

Figure 3 : Overall concent

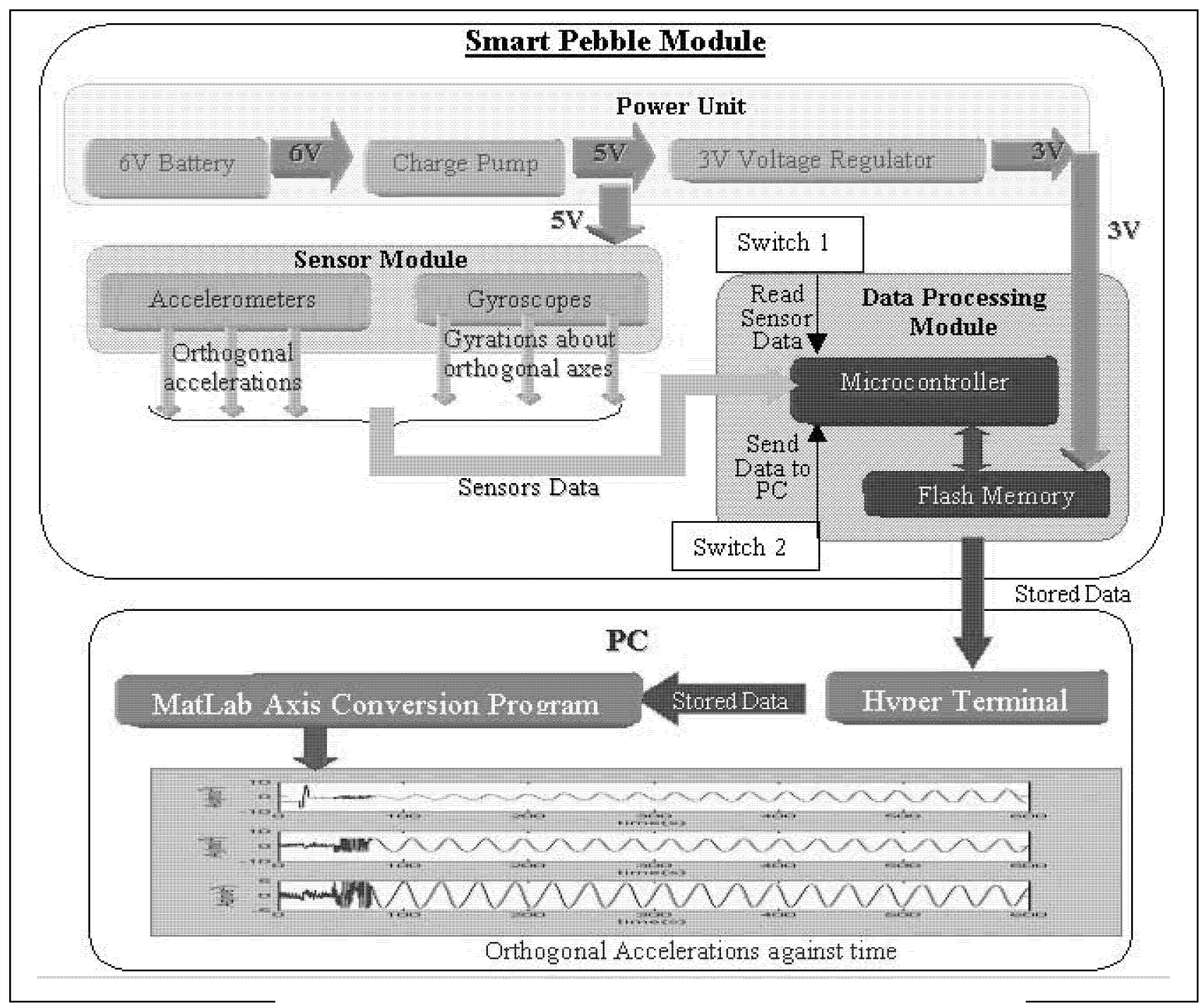

Figure 4: Total system block diagram including off-line Matlab 
The unit is powered by a $6 \mathrm{~V}$ battery, which generally powers the circuit up to about 15 minutes continuously. A Texas Instruments TPS60132 charge pump has been utilized to regulate the supply voltage of the pebble at $5 \mathrm{~V}$. Additionally, it boosts the battery voltage as it dips below $5 \mathrm{~V}$ to maintain the accuracy of the sensor readings. The entire system works on $5 \mathrm{~V}$, except for the external flash memory which requires $3 \mathrm{~V}$ supplied through a REG102 3V regulator.

Since dual axis accelerometers were used, it was possible to take all the necessary measurements with two chips to keep the real estate of the PCBs to a minimum. Each sensor produces an analogue voltage proportional to the acceleration or gyration measured. These outputs are periodically scanned and averaged out to minimize noise, through the analogue-to-digital-converter channels of the Microchip PIC18F8520 microcontroller. The digitized sensor data are then stored in an external flash memory, SST25VF020. Since the data are collected at regular time intervals, these data are later processed to determine the motion of the pebble.

The sensors are mounted such that the accelerations along the three orthogonal axes and the gyrations about each axis are measured. Figure 5 depicts the orientation of the sensors and their sensitive axes. It can be observed that the sensors actually measure accelerations and gyrations about the $-\mathrm{y}$-axis as opposed to the $y$-axis. These measurements are later processed in MATLAB, to obtain the measurements about the $y$-axis.

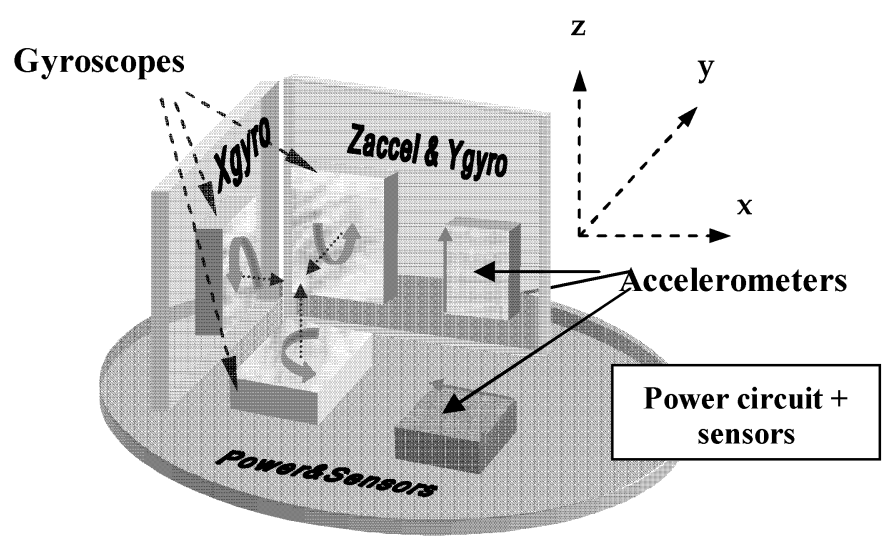

Figure 5: Orientation of sensors of the complete system

The smart pebble is a complete, battery powered unit equipped with two dual-axis accelerometers and three angular rate sensors, a microcontroller, non-volatile external memory and a user interface capable of communication with a PC. It has been packaged into a sphere of $3 \mathrm{~cm}$ diameter as illustrated in Figure 6.

The stored data are transferred to a PC over the hyper terminal of the computer. These data are then processed in MATLAB to calculate the true accelerations along the reference axes. The MATLAB program takes in the raw digitized sensor data and converts them to the analogue voltage values measured originally. Since the fixed axis set of the sensor boards are subject to rotations, the accelerations measured are, in fact, not the true accelerations along a set of reference axes. Therefore, the data need complex mathematical processing to extract the true acceleration data as per details in section II.

First, the gyroscope measurements are integrated to obtain the rotation angles of the unit, about each axis. Next, the initial tilt of the unit and the gravity components along each axis are calculated. Then the direction cosine matrix and Euler angles are used to convert the data to obtain the true accelerations along each reference axis. Finally gravity is compensated for and the accelerations along the reference axes are plotted.

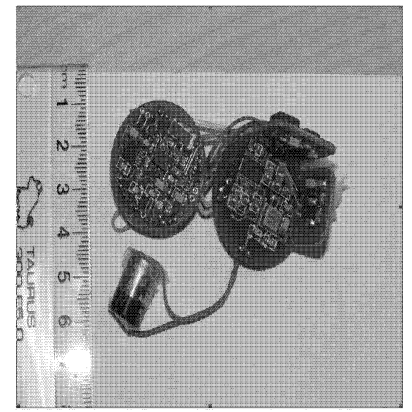

(a)

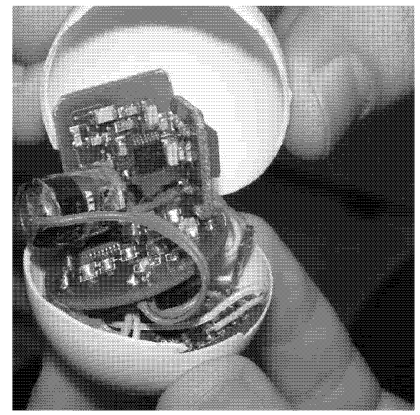

(b)
Figure 6: Final packaged 'Smart Pebble'. (a) Actual size of the PCBs. (b) The PCBs inside the shell of the smart pebble

\section{CALIBRATION AND TESTING}

In developing a system of this nature, the designer needs to be aware of the overall errors contributed by many sources. Some obvious errors are the accelerometer offsets, OpAmp offsets, the analog calculation interface inaccuracies and offsets and noise from the sensors. The unit was mounted on a small trolley placed on a variable slope and then attached to a string coupled to a digital shaft encoder for calibration as shown in Figure 7. Individual sensor output data from the system are filtered using digital filters and then compared with the shaft encoder readings as shown in Figure 8.

The graphs in Figure 9 indicate the measurements down the slope over an approximate period of 0.5 seconds, indicating the relationships between some representative acceleration readings from the pebble and the shaft encoder measurements. Normalized motion parameters plotted with shaft encoder accelerations along each axis indicate that there was a reasonable correlation among the sets of readings. 


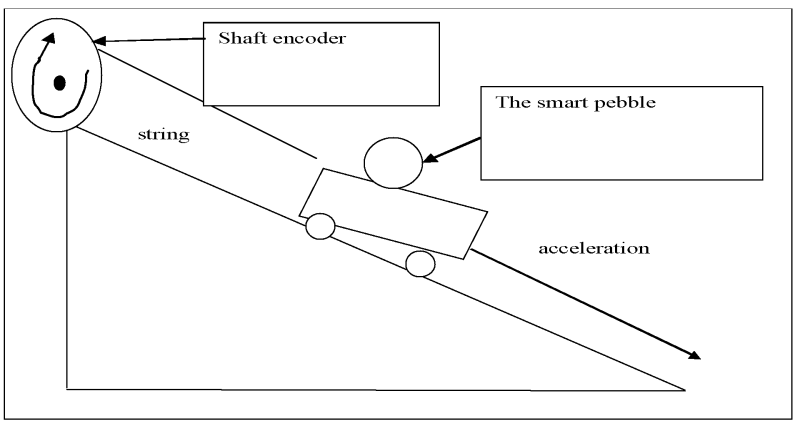

Figure 7: Side view of calibrating the system with the shaft encoder.

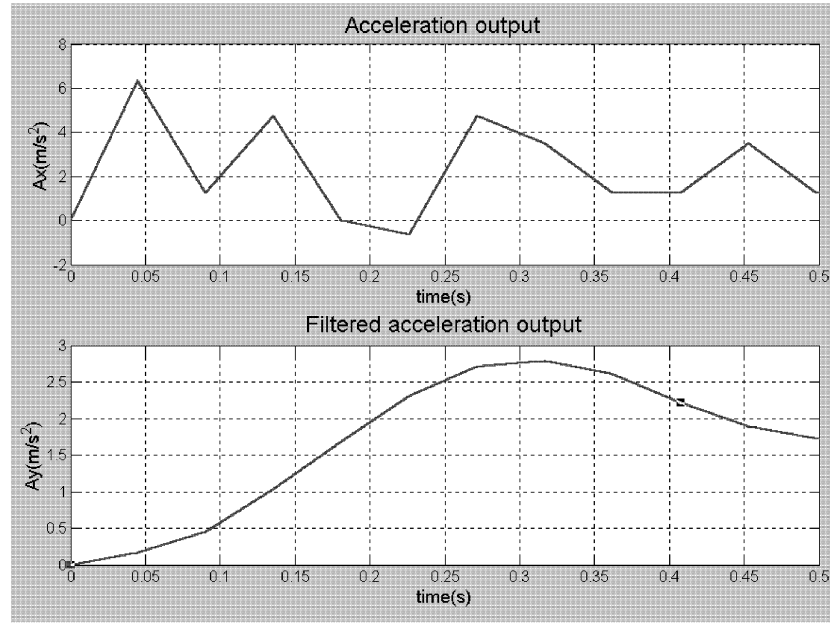

Figure 8: Filtering acceleration output.

\section{CONCLUSION}

The paper describes the total implementation aspects of a smart pebble usable for monitoring of the 3-D motion parameters of the pebble, including the rotations around 3 axes. The possibility of autonomous operation with a battery based energy source for storing and extracting raw data into an off-line processing system is covered. The design allows running of the total system for over 10 minutes with a single alkaline cell as shown in the Figure 6 . A description of the details of the Matlab program to convert the raw body frame data to reference axes and the gravity compensation, etc, are not covered in this paper. This system approach and the implementation details clearly indicate that due to very short flight times expected in the civil engineering application, low cost MEMS are usable. Further work is in progress.

\section{ACKNOWLEDGMENT}

This project was funded by the Department of Civil and Environmental Engineering, University of Auckland. The authors appreciate the help of the technical staff members, Geoff Kirby and Gary Carr of the civil engineering laboratories, in setting up the calibration system. The assistance given by the civil engineering postgraduate student Ambuj Dwivedi, during the latter stages of the work, is also acknowledged.

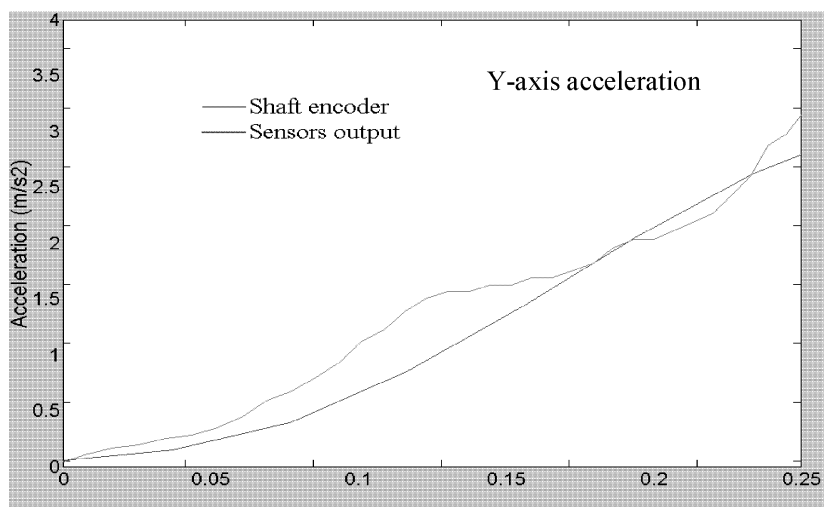

Time (s)

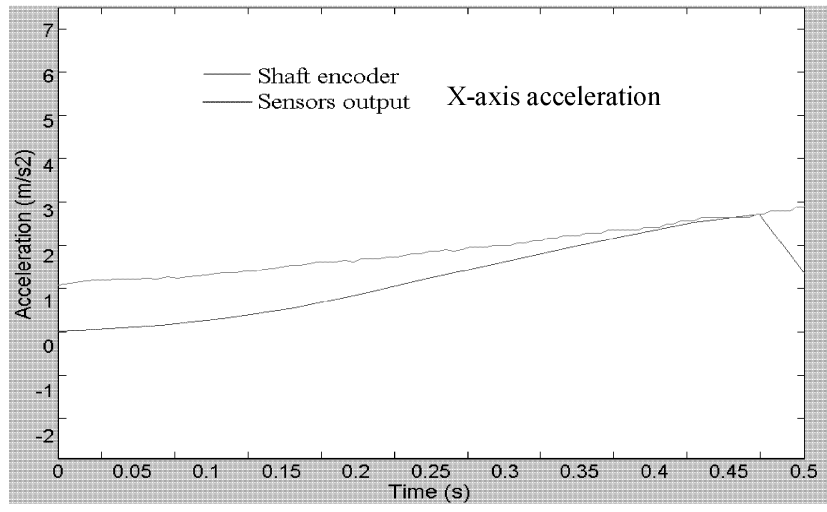

Figure 9: Testing the final system readings with the readings

\section{REFERENCES}

1. Kularatna, N. Melville, B. and Wijeratne, C. "Mixed Signal Approach for Rapid Prototyping of a Compact Smart Pebble for Sediment Transport Monitoring in River Beds", 2005 IEEE Sensors, Oct 2005, pp 1128 - 1132

2. Brown, T.G et el; " Strap-down micromechanical (MEMS) sensors for high-G munition applications"; IEEE Trans on Magnetics, Vol. 37, No 1, Jan 2001

3. Tieman, J. et el; " Design of a MEMS based 3-axis accelerometer smart sensor"; Proc of IEEE Sensors for Industry Conf, USA, Nov 2002, pp 19-23.

4. Coleman, S.E. and Melville, B.W. "Initiation of Bed Forms on a Flat Sand Bed", Journal of Hydraulic Engineering, ASCE, 122 (6), 301-310, June, 1996.

5. Garcia, M.H., Lopez, F. and Nino, Y. "Characterization of near-bed coherent structures in open channel flow using synchronized high-speed video and hot film measurements," Experiments in Fluids, Vol. 19, 16-28, 1995

6. Titerron, D.H. \& Weston, J.L.: " Strapdown inertial navigation technology"; IEE, 1997

7. Pio, R:"Euler angle transformations"; IEEE Transactions on Automatic Control, Volume 11, Issue 4, Oct 1966 Page(s):707 - 715 